PROCEEDINGS OF THE

AMERICAN MATHEMATICAL SOCIETY

Volume 139, Number 10, October 2011, Pages 3485-3490

S 0002-9939(2011)10973-0

Article electronically published on June 6, 2011

\title{
LOGARITHMIC UP BOUNDS FOR SOLUTIONS OF ELLIPTIC PARTIAL DIFFERENTIAL EQUATIONS
}

\author{
XIANGSHENG XU \\ (Communicated by Walter Craig)
}

\begin{abstract}
In this paper we obtain a logarithmic up bound for the solution to an elliptic mixed boundary value problem. This is an improvement over the corresponding classical result in this area.
\end{abstract}

\section{MAin ReSult AND PROOF}

Let $\Omega$ be a bounded domain in $R^{N}, N>2$, with Lipschitz boundary $\partial \Omega$. Assume that $\Gamma_{D}$ is a non-empty open subset of $\partial \Omega$. Consider the mixed boundary value problem

$$
\begin{aligned}
-\operatorname{div}(A(x) \nabla \varphi) & =f \text { in } \Omega, \\
\varphi & =\varphi_{0} \text { on } \Gamma_{D}, \\
A(x) \nabla \varphi \cdot \nu & =0 \text { on } \Gamma_{N} \equiv \partial \Omega \backslash \overline{\Gamma_{D}} .
\end{aligned}
$$

Here $A(x)$ is an $N \times N$ matrix whose entries $a_{i j}(x)$ satisfy

$$
a_{i j} \in L^{\infty}(\Omega), \quad \lambda|\xi|^{2} \leq A(x) \xi \cdot \xi \quad \text { for } \quad \xi \in R^{N} \quad \text { a.e. } \quad x \in \Omega,
$$

where $\lambda>0$. As for other given data in the problem we have that $f \in L^{\frac{p}{2}}(\Omega)$ for some $p>N, \varphi_{0} \in W^{1,2}(\Omega) \cap L^{\infty}(\Omega)$, and $\nu$ is the unit outward normal to $\partial \Omega$. In the situation considered here, the classical theory for uniformly elliptic equations asserts that there is a unique weak solution $\varphi$ in the space $W^{1,2}(\Omega)$ and that $\varphi$ satisfies

$$
\|\varphi\|_{\infty} \leq\left\|\varphi_{0}\right\|_{\infty}+c\|f\|_{\frac{p}{2}}, \quad c=c\left(\lambda, p, \Gamma_{D}, \Omega\right) .
$$

Here and in what follows we denote by $\|f\|_{p}$ the norm of $f$ in $L^{p}(\Omega)$. The result is sharp in the sense that if $p=N$, then (1.5) fails in general. The objective of this paper is to improve the up bound for $\|\varphi\|_{\infty}$. To be precise, we have shown:

Theorem A. Under the above assumptions there is a positive number $c=$ $c\left(\lambda, p, \Gamma_{D}, \Omega\right)$ such that

$$
\|\varphi\|_{\infty} \leq\left\|\varphi_{0}\right\|_{\infty}+c\|f\|_{\frac{N}{2}}\left(\ln \left(\|f\|_{\frac{p}{2}}+1\right)+1\right) .
$$

Received by the editors July 7, 2010 and, in revised form, July 13, 2010.

2010 Mathematics Subject Classification. Primary 35B50, 35D30; Secondary 35J67, 35J15.

Key words and phrases. Logarithmic up bound, mixed boundary value problem.

(C)2011 American Mathematical Society Reverts to public domain 28 years from publication 
A similar result for functions in $W^{s, p}\left(R^{N}\right), s p>N$, is established in 1, 2].

To prove Theorem A, we decompose $\varphi$ into $\varphi_{1}+\varphi_{2}$, where $\varphi_{1}$ and $\varphi_{2}$ are the respective solutions of the following two problems:

$$
\begin{aligned}
-\operatorname{div}\left(A(x) \nabla \varphi_{1}\right) & =f \text { in } \Omega, \\
\varphi_{1} & =0 \text { on } \Gamma_{D}, \\
A(x) \nabla \varphi_{1} \cdot \nu & =0 \text { on } \Gamma_{N}
\end{aligned}
$$

and

$$
\begin{aligned}
-\operatorname{div}\left(A(x) \nabla \varphi_{2}\right) & =0 \text { in } \Omega, \\
\varphi_{2} & =\varphi_{0} \text { on } \Gamma_{D}, \\
A(x) \nabla \varphi_{2} \cdot \nu & =0 \text { on } \Gamma_{N} .
\end{aligned}
$$

Now set

$$
\begin{aligned}
u & =\frac{\varphi_{1}}{\|f\|_{\frac{N}{2}}}, \\
g & =\frac{f}{\|f\|_{\frac{N}{2}}} .
\end{aligned}
$$

Then we have

$$
\begin{aligned}
-\operatorname{div}(A(x) \nabla u) & =g \text { in } \Omega, \\
u & =0 \text { on } \Gamma_{D}, \\
A(x) \nabla u \cdot \nu & =0 \text { on } \Gamma_{N} .
\end{aligned}
$$

Lemma B. Let $c_{s}$ be the smallest positive number such that

$$
\|\phi\|_{2^{*}} \leq c_{s}\|\nabla \phi\|_{2}
$$

for all $\phi \in W^{1,2}(\Omega)$ with $\phi=0$ on $\Gamma_{D}$. It is well-known that the Sobolev constant $c_{s}$ here depends only on $\Gamma_{D}, \Omega$. To each $\alpha \in\left(0, \frac{4 \lambda}{c_{s}^{2}}\right)$ there corresponds a positive number $c$ such that

$$
\int_{\Omega} \exp \left(\frac{\alpha N u}{N-2}\right) d x \leq c
$$

Proof. Let $\alpha \in\left(0, \frac{4 \lambda}{c_{s}^{2}}\right)$ be given. Since $u$ is bounded, $e^{\alpha u}-1$ is a legitimate test function for (1.15), and upon using it, we obtain

$$
\lambda \alpha \int_{\Omega} e^{\alpha u}|\nabla u|^{2} d x \leq \alpha \int_{\Omega} A(x) \nabla u \cdot e^{\alpha u} \nabla u d x=\int_{\Omega} g\left(e^{\alpha u}-1\right) d x .
$$


Due to the Sobolev Imbedding Theorem and the fact that $e^{\alpha u}-1=\left(e^{\frac{\alpha u}{2}}-1\right)^{2}+$ $2\left(e^{\frac{\alpha u}{2}}-1\right)$, we estimate

$$
\begin{aligned}
\left(\int_{\Omega}\left(e^{\frac{\alpha u}{2}}-1\right)^{\frac{2 N}{N-2}} d x\right)^{\frac{N-2}{N}} \leq & c_{s}^{2} \int_{\Omega}\left|\nabla\left(e^{\frac{\alpha u}{2}}-1\right)\right|^{2} d x \\
& =\frac{c_{s}^{2} \alpha^{2}}{4} \int_{\Omega} e^{\alpha u}|\nabla u|^{2} d x \\
\leq & \frac{c_{s}^{2} \alpha}{4 \lambda} \int_{\Omega} g\left(e^{\alpha u}-1\right) d x \\
= & \frac{c_{s}^{2} \alpha}{4 \lambda} \int_{\Omega} g\left(e^{\frac{\alpha u}{2}}-1\right)^{2} d x+\frac{c_{s}^{2} \alpha}{2 \lambda} \int_{\Omega} g\left(e^{\frac{\alpha u}{2}}-1\right) d x \\
\leq & \frac{c_{s}^{2} \alpha}{4 \lambda}\|g\|_{\frac{N}{2}}\left(\int_{\Omega}\left(e^{\frac{\alpha u}{2}}-1\right)^{\frac{2 N}{N-2}} d x\right)^{\frac{N-2}{N}} \\
& +\frac{c_{s}^{2} \alpha}{2 \lambda}\|g\|_{\frac{2 N}{N+2}}\left(\int_{\Omega}\left(e^{\frac{\alpha u}{2}}-1\right)^{\frac{2 N}{N-2}} d x\right)^{\frac{N-2}{2 N}} .
\end{aligned}
$$

Take into consideration $1-\frac{c_{s}^{2} \alpha}{4 \lambda}>0$ and (1.14) to deduce

$$
\left(\int_{\Omega}\left(e^{\frac{\alpha u}{2}}-1\right)^{\frac{2 N}{N-2}} d x\right)^{\frac{N-2}{2 N}} \leq \frac{2 c_{s}^{2} \alpha\|g\|_{\frac{2 N}{N+2}}}{4 \lambda-c_{s}^{2} \alpha} .
$$

Note that

$$
\|g\|_{\frac{2 N}{N+2}}=\frac{\|f\|_{\frac{2 N}{N+2}}}{\|f\|_{\frac{N}{2}}} \leq|\Omega|^{\frac{N-2}{2 N}} .
$$

We arrive at

$$
\begin{aligned}
\left\|e^{\frac{\alpha u}{2}}\right\|_{\frac{2 N}{N-2}} & \leq\left\|e^{\frac{\alpha u}{2}}-1\right\|_{\frac{2 N}{N-2}}+|\Omega|^{\frac{N-2}{2 N}} \\
& \leq \frac{2 c_{s}^{2} \alpha|\Omega|^{\frac{N-2}{2 N}}}{4 \lambda-c_{s}^{2} \alpha}+|\Omega|^{\frac{N-2}{2 N}} \\
& =\frac{4 \lambda+c_{s}^{2} \alpha}{4 \lambda-c_{s}^{2} \alpha}|\Omega|^{\frac{N-2}{2 N}}
\end{aligned}
$$

The proof of the lemma is complete.

To continue the proof of Theorem A, we assume without any loss of generality that

$$
\begin{aligned}
\operatorname{ess} \sup _{\Omega} u & =\|u\|_{\infty} \\
\|f\|_{\frac{N}{2}} & \geq 1 .
\end{aligned}
$$

Indeed, if (1.22) is not true, we multiply through (1.15) by -1 and consider $-u$. If (1.23) is not true, we consider the problem (1.7)-(1.9) directly because we already have that (1.19) holds for $u=\varphi_{1}$. Let

$$
v=e^{u} .
$$


Then

$$
\begin{aligned}
u & =\ln v \\
A(x) \nabla u & =\frac{1}{v} A(x) \nabla v, \\
\operatorname{div}(A(x) \nabla u) & =-\frac{1}{v^{2}} A(x) \nabla v \cdot \nabla v+\frac{1}{v} \operatorname{div}(A(x) \nabla v) .
\end{aligned}
$$

Substitute these into (1.15)-(1.17) to obtain

$$
\begin{aligned}
-\operatorname{div}(A(x) \nabla v)+\frac{1}{v} A(x) \nabla v \cdot \nabla v & =g v \text { in } \Omega, \\
v & =1 \text { on } \Gamma_{D}, \\
A(x) \nabla v \cdot \nu & =0 \text { on } \Gamma_{N} .
\end{aligned}
$$

The above formal calculations can easily be made vigorous. Set

$$
w=(v-1)^{+}+1 \text {. }
$$

Then it is easy to see that

$$
w(x)= \begin{cases}v(x) & \text { if } v(x)>1 \\ 1 & \text { if } v(x) \leq 1\end{cases}
$$

For $\beta>0$, we use $w^{\beta}-1$ as a test function in (1.25) to obtain

$$
\int_{\Omega} \beta w^{\beta-1} A(x) \nabla v \cdot \nabla w d x \leq \int_{\Omega} g v\left(w^{\beta}-1\right) d x .
$$

This together with (1.28) implies

$$
\lambda \beta \int_{\Omega} w^{\beta-1}|\nabla w|^{2} d x \leq \int_{\Omega} g v\left(w^{\beta}-1\right) d x \leq \int_{\Omega}|g| w^{\beta+1} d x .
$$

Note that

$$
\begin{aligned}
w^{\beta+1} & =\left(w^{\frac{\beta+1}{2}}-1+1\right)^{2} \\
& \leq 2\left(w^{\frac{\beta+1}{2}}-1\right)^{2}+2 .
\end{aligned}
$$

Keeping this in mind and with the aid of the Sobolev Imbedding Theorem and (1.30), we calculate

$$
\begin{aligned}
\left\|w^{\beta+1}\right\|_{\frac{N}{N-2}} & \leq 2\left\|\left(w^{\frac{\beta+1}{2}}-1\right)^{2}\right\|_{\frac{N}{N-2}}+2|\Omega|^{\frac{N-2}{N}} \\
& \leq c_{s}^{2} \frac{(\beta+1)^{2}}{2} \int_{\Omega} w^{\beta-1}|\nabla w|^{2} d x+2|\Omega|^{\frac{N-2}{N}} \\
& =\frac{c(\beta+1)^{2}}{\beta} \int_{\Omega}|g| w^{\beta+1} d x+2|\Omega|^{\frac{N-2}{N}},
\end{aligned}
$$

where $c=c\left(c_{s}, \lambda\right)=c\left(\Gamma_{D}, \Omega, \lambda\right)$. Remember $w \geq 1$ on $\Omega$. For $p>N$, we have

$$
\begin{aligned}
\left\|w^{\beta+1}\right\|_{\frac{N}{N-2}} & \leq \frac{c(\beta+1)^{2}}{\beta}\|g\|_{\frac{p}{2}}\left(\int_{\Omega} w^{\frac{(\beta+1) p}{p-2}} d x\right)^{\frac{p-2}{p}}+2\left(\int_{\Omega} w^{\frac{(\beta+1) p}{p-2}} d x\right)^{\frac{p-2}{p}}|\Omega|^{\frac{2}{p}-\frac{2}{N}} \\
& =\left(\frac{c(\beta+1)^{2}}{\beta}\|g\|_{\frac{p}{2}}+2|\Omega|^{\frac{2}{p}-\frac{2}{N}}\right)\left\|w^{\beta+1}\right\|_{\frac{p}{p-2}} .
\end{aligned}
$$


Observe that

$$
\frac{(\beta+1)^{2}}{\beta}>4
$$

Thus we can conclude that

$$
\|w\|_{\frac{N(\beta+1)}{N-2}} \leq\left[c \frac{(\beta+1)^{2}}{\beta}\left(\|g\|_{\frac{p}{2}}+1\right)\right]^{\frac{1}{\beta+1}}\|w\|_{\frac{p(\beta+1)}{p-2}} .
$$

Now set

$$
\chi=\frac{N /(N-2)}{p /(p-2)}>1 .
$$

Fix $\beta_{0}>0$ and let

$$
\beta+1=\left(1+\beta_{0}\right) \chi^{i}, \quad i=0,1,2, \cdots .
$$

Subsequently,

$$
\begin{aligned}
\|w\|_{\left(1+\beta_{0}\right) \frac{p}{p-2}} \chi^{i+1} \leq & \left(\frac{c}{\beta_{0}}\left(\|g\|_{\frac{p}{2}}+1\right)\right)^{\frac{1}{\left(1+\beta_{0}\right) \chi^{i}}}\left[\left(1+\beta_{0}\right)^{2} \chi^{2 i}\right]^{\frac{1}{\left(1+\beta_{0}\right) \chi^{i}}}\|w\|_{\left(1+\beta_{0}\right) \frac{p}{p-2} \chi^{i}} \\
\leq & {\left[c\left(\|g\|_{\frac{p}{2}}+1\right) \frac{\left(1+\beta_{0}\right)^{2}}{\beta_{0}}\right]^{\frac{1}{\left(1+\beta_{0}\right)}\left(\frac{1}{\chi^{i}}+\cdots+1\right)} } \\
& \cdot \chi^{\frac{2}{\left(1+\beta_{0}\right)}\left(\frac{i}{\chi^{i}}+\cdots+1\right)}\|w\|_{\left(1+\beta_{0}\right) \frac{p}{p-2}} .
\end{aligned}
$$

Taking $i \rightarrow \infty$ yields

$$
\|w\|_{\infty} \leq c\left(\|g\|_{\frac{p}{2}}+1\right)^{\alpha_{0}}\|w\|_{\left(1+\beta_{0}\right) \frac{p}{p-2}}
$$

where $\alpha_{0}$ is a positive number depending on $\beta_{0}, p$. Let

$$
q=\left(1+\beta_{0}\right) \frac{p}{p-2} .
$$

We can assume that

$$
q<\frac{4 \lambda N}{c_{s}^{2}(N-2)} .
$$

If not, pick a number $\alpha$ so that

$$
0<\alpha<\frac{4 \lambda N}{c_{s}^{2}(N-2)} \leq q .
$$

Then we have

$$
\begin{aligned}
\|w\|_{q} & =\left(\int_{\Omega} w^{q-\alpha} w^{\alpha} d x\right)^{\frac{1}{q}} \\
& \leq\|w\|_{\infty^{\frac{q-\alpha}{q}}}^{q}\|w\|_{\alpha}^{\frac{\alpha}{q}} .
\end{aligned}
$$

We derive from (1.33) that

$$
\begin{aligned}
\|w\|_{\infty} & \leq c\left(\|g\|_{\frac{p}{2}}+1\right)^{\alpha_{0}}\|w\|_{\infty}^{\frac{q-\alpha}{q}}\|w\|_{\alpha}^{\frac{\alpha}{q}} \\
& \leq \frac{1}{2}\|w\|_{\infty}+c\left(\|g\|_{\frac{p}{2}}+1\right)^{\alpha_{0} \frac{q}{\alpha}}\|w\|_{\alpha}
\end{aligned}
$$

from whence it follows that

$$
\|w\|_{\infty} \leq c\left(\|g\|_{\frac{p}{2}}+1\right)^{\alpha_{0} \frac{q}{\alpha}}\|w\|_{\alpha} .
$$


Recall the definition of $w$ to obtain

$$
\|v\|_{\infty} \leq c\left(\|g\|_{\frac{p}{2}}+1\right)^{\alpha_{0} \frac{q}{\alpha}}\left(\|v\|_{\alpha}+|\Omega|^{\frac{1}{\alpha}}\right) .
$$

In view of (1.24), (1.34), (1.22) and (1.19), we obtain

$$
e^{\|u\|_{\infty}} \leq c\left(\|g\|_{\frac{p}{2}}+1\right)^{\alpha_{0} \frac{q}{\alpha}} .
$$

Take the logarithm and make a note of (1.23) to get

$$
\|u\|_{\infty} \leq c\left(\ln \left(\|f\|_{\frac{p}{2}}+1\right)+1\right) .
$$

Finally, we derive

$$
\begin{aligned}
\|\varphi\|_{\infty} & \leq\left\|\varphi_{1}\right\|_{\infty}+\left\|\varphi_{2}\right\|_{\infty} \\
& \leq c\|f\|_{\frac{N}{2}}\left(\ln \left(\|f\|_{\frac{p}{2}}+1\right)+1\right)+\left\|\varphi_{0}\right\|_{\infty} .
\end{aligned}
$$

This completes the proof.

\section{REFERENCES}

[1] H. Brezis and S. Wainger, A note on limiting cases of Sobolev embedding and convolution inequalities, Comm. Partial Differential Equations, 5(1980), pp. 773-789. MR579997|(81k:46028)

[2] H. Kozono and Y. Taniuchi, Limiting case of the Sobolev inequality in BMO with application to the Euler equations, Commun. Math. Phys., 214(2000), pp. 191-200. MR.1794270 (2002k:46081)

Department of Mathematics and Statistics, Mississippi State University, Mississippi State, Mississippi 39762 